\title{
Valoración del Informe Pericial sobre la Custodia de Menores en Sentencias Judiciales: Estudio comparativo entre informes privados y oficiales
}

\section{Expert Assessment Report on Child Custody in Court Judgments: Comparative study between private and official reports}

\author{
Carles Rodríguez-Domínguez ${ }^{1}$ y Adolfo Jarne Espacia ${ }^{2}$ \\ ${ }^{1}$ FPCEE Blanquerna. Universidad Ramón Llull, España \\ ${ }^{2}$ Universidad de Barcelona, España
}

Disponible online 31 de diciembre de 2015

\begin{abstract}
En España, el divorcio con menores involucrados ha comportado un incremento sustancial de demandas de informes periciales. El objetivo ha sido analizar la valoración en sentencias judiciales de los informes periciales privados con formación específica forense, informes periciales privados sin formación específica forense e, informes periciales de los equipos psicosociales de los Juzgados sobre la guarda y custodia y el régimen de visitas del progenitor no custodio. Se aplicó un protocolo confeccionado ad hoc por los autores a 111 expedientes contenciosos de los Juzgados de Familia. Se hallaron diferencias significativas en las valoraciones expresadas por las sentencias judiciales sobre los informes periciales privados y los informes periciales de los equipos psicosociales. Los análisis sugieren que las sentencias expresaron una opinión favorable con preferencia por los informes periciales realizados por los equipos psicosociales adscritos a los juzgados de familia. Se aporta evidencia empírica directa. Un $56.8 \%$ de las sentencias expresaron una opinión favorable de los informes periciales, e instaron a las partes a seguir las recomendaciones del informe pericial.
\end{abstract}

Palabras Clave: Informe Pericial Psicológico; Divorcio Conflictivo; Guarda y Custodia de Menores; Valoración del Informe en Sentencias.

In Spain, divorce with children involved has led to a substantial increase in demand for expert reports. The aim was to analyse the valuation judgments of private forensic expert reports with specific training, private forensic expert reports without specific training and, expert reports of psychosocial teams of the Courts on the custody and visitation of the parent no custodian. Ad hoc protocol made by the authors to 111 litigation files of Family Courts was applied. Significant differences in the evaluations expressed by judicial decisions on private expert reports and the expert reports of psychosocial teams were found. Analysis suggests that the judgments expressed a favourable opinion with preference for expert reports made by employees assigned to family courts psychosocial teams. Direct empirical evidence is provided. $56.8 \%$ of the judgments expressed a favourable opinion of the expert reports and urged the parties to follow the recommendations of the expert report.

Key Words: Psychological Expert Report; Contentious Divorce; Guardianship and Custody of Minors; Evaluation Reports Sentences.

Correspondencia: Carles Rodríguez Domínguez. FPCEE. c/ Císter 34, Blanquerna. 08022- Barcelona. E-mail: carlesrd@blanquerna.url.edu. E-mail del co-autor: Adolfo Jarne Espacia: ajarne@ub.edu.

Los autores expresan su agradecimiento al Tribunal Superior de Justicia de Catalunya y a los Secretarios Judiciales responsables de la custodia de los archivos que nos autorizaron para la realización del estudio de los expedientes contenciosos en los Juzgados de Familia 15, 16, 17, 18, 19, 45, y 51 de Barcelona. 
La función pericial en España está regulada en Derecho Civil por la Ley de Enjuiciamiento Civil. Los peritos son profesionales con conocimientos especializados citados al proceso judicial para aportar distintos conocimientos técnicos que el Juez no tiene por qué poseer. Conocimientos técnicos necesarios para valorar hechos que no podrían lograrse sin tal pericia (Ley Enjuiciamiento Civil, 1/2000). El informe pericial se realiza durante La fase de obtención de la prueba; es un procedimiento legal a disposición del Juez para su labor en las decisiones judiciales, tal como recoge el Art. 335 de la Ley Enjuiciamiento Civil. El experto en su función pericial no está sujeto al secreto profesional respecto al objeto de la pericia en el contexto jurídico, por consiguiente ha de comunicarlo a los sujetos evaluados y requerir su consentimiento informado antes de iniciar el peritaje (Echeburúa, 2002).

Las características esenciales del informe pericial forense deben mantener la estructura y la metodología recomendada por las directrices de la American Psychological Association (APA) y de orientaciones éticas de los colegios profesionales de psicólogos y de los trabajadores sociales (APA, 1994, 2009, 2010a , 2010b, 2012; CGTS, 2012; COP, 2007; COPM, 2009). Legalmente, el contenido del informe pericial está insuficientemente regulado, no obstante, debe ajustarse al objeto de la demanda judicial y contemplar exclusivamente la información relevante para la finalidad de la evaluación (Urra, 2007). Al ser considerado un medio probatorio está sujeto al principio de contradicción, por cuya razón, ambas partes pueden examinarlo, criticarlo o censurarlo mediante un contra informe. Debe exponer la información analizada por la que llegó a las conclusiones descritas, así como los argumentos científicos utilizados para avalar los datos técnicos. Debe especificar la metodología empleada, las fuentes de información contrastadas, los instrumentos técnicos utilizados y los resultados de las pruebas administradas (Del Río, 2005). Es un documento dinámico, válido y referido al momento de la exploración puesto que las circunstancias pueden cambiar. Se ha de ratificar en la vista oral para explicar, clarificar o ampliar los datos pertinentes solicitados por los letrados o el tribunal. La Autoridad Judicial puede solicitar los documentos de las pruebas aplicadas. Alcanza mayor notabilidad cuando relaciona la metodología aplicada con el estado actual del conocimiento científico; cuando establece un vínculo claro entre la evaluación pericial y el asunto contencioso planteado en la demanda judicial; sin deformar la información lograda; y si es escrito con un lenguaje claro, riguroso y exento de tecnicismos innecesarios (Esbec y Gómez- Jarabo, 2000; Illescas, 2005).

En el entorno anglosajón, varios estudios han destacado numerosas carencias sobre el conocimiento real de los informes psicológicos periciales; además, se ha señalado la necesidad de modelos estandarizados de informe (Martindale et al., 2007); se ha cuestionado la variedad de técnicas utilizadas y se ha criticado la incoherencia entre las directrices de la APA y la práctica clínico-forense (Tippins y Wittmann, 2005).
Por otra parte, cabe advertir al lector, la salvedad de que no pueden compararse directamente los estudios anglosajones con los del entorno español por: a) proceder de muestras distintas, y b) por considerar la cautela necesaria de que la legislación estadounidense muy estricta con sus operadores jurídicos, difiere de nuestro sistema legislativo, si bien se entiende que estos estudios puede, ser de utilidad como marco de referencia.

Un estudio realizado en 38 estados de EEUU (Bow y Quinnell, 2001), sobre una muestra de 279 evaluadores de la custodia de menores, en relación a la congruencia entre las directrices de la APA y las prácticas de las evaluaciones forenses, así como los cambios en custodia de los hijos en el período 1986-2001, concluyó un progreso hacia una mayor formación profesional obtenida en relación con las directrices APA pero, hay que resaltar, que en este estudio el $96.0 \%$ de los encuestados eran doctores en psicología con formación forense.

En otro estudio, Symons (2010) planteó la falta de evidencia empírica directa de las evaluaciones de la custodia de menores, considerada como una de las áreas trascendentales de investigación que incluye los efectos de la separación y el divorcio en los niños, las relaciones familiares, y el desarrollo de los procesos. Asimismo, Kelly y Ramsey (2009) criticaron el estado actual de la investigación sobre las evaluaciones de custodia pese a la dificultad de acceso a los registros de los juzgados para realizar estudios. La evaluación de la custodia de menores aspira a sustentarse en los principios científicos de la práctica profesional, por lo que hay una obligación profesional de cumplir con los conocimientos probados en los que dicha praxis se fundamenta.

Otro estudio realizado sobre el análisis de contenidos de casos en las evaluaciones de la custodia de menores en la práctica (Horvath, Logan y Walker, 2002), concluyó que existía una gran variedad de técnicas utilizadas y una falta de coherencia entre las directrices y la práctica clínica. Según los autores, estos hallazgos sugieren la necesidad de enfoques más estandarizados para la realización de evaluaciones de custodia de menores, así como de la evaluación de la violencia doméstica, el abuso infantil y del abuso de sustancias.

El marco de intervención de la evaluación de la custodia de menores imprime las pautas de relación (Ackerman, 2010; Echeburúa, Muñoz y Loínaz, 2011) entre el evaluador privado y el progenitor que realiza la demanda con el frecuente escepticismo y reservas del otro progenitor litigante. En el cuadro 1 recogemos algunos conceptos positivos y conceptos a considerar referidos a las evaluación de la custodia de menores de los profesionales privados y públicos.

En un estudio sobre el nivel de conocimiento de ética deontológica de los psicólogos forenses expertos en el ámbito de familia, ninguna de las situaciones presentadas obtuvo una respuesta ética unánime de los participantes (Molina, Arch y Jarne, 2012). Las consideraciones atribuyeron un carácter exagera- 
Cuadro 1

Evaluación pericial privada y evaluación pericial del Equipo Psicosocial: diferencias a valorar.

\begin{tabular}{|c|c|c|c|c|}
\hline \multirow{2}{*}{ Conceptos } & \multicolumn{2}{|c|}{ Evaluación pericial Privada } & \multicolumn{2}{|c|}{ Evaluación Pericial del Equipo Psicosocial } \\
\hline & Aspecto Positivo & A considerar & Aspecto Positivo & A considerar \\
\hline Cliente & $\begin{array}{l}\text {-Demanda del usuario } \\
\text { Evaluación de los menores. }\end{array}$ & $\begin{array}{l}\text {-Posible parcialidad } \\
\text { Múltiples criterios. } \\
\text { Contra informes. } \\
\text { Mayor dificultad de evaluar } \\
\text { al otro progenitor por } \\
\text { escepticismo natural del litigio. }\end{array}$ & $\begin{array}{l}\text {-Citación Judicial. } \\
\text { Criterios especificados por } \\
\text { el Juez. } \\
\text { Suelen acudir ambos } \\
\text { progenitores. }\end{array}$ & $\begin{array}{l}\text { Contra informes. } \\
\text { Insuficiente evaluación de } \\
\text { menores. }\end{array}$ \\
\hline Críticas recibidas & $\begin{array}{l}\text {-Evaluación de menores. } \\
\text { Más uso de pruebas } \\
\text { psicométricas. }\end{array}$ & $\begin{array}{l}\text {-Menos valorados } \\
\text { positivamente por los Jueces } \\
\text { por posibilidad de parcialidad. } \\
\text { Insuficiente estandarización } \\
\text { Protocolos. }\end{array}$ & $\begin{array}{l}\text {-Valorados positivamente por } \\
\text { los Jueces. } \\
\text { Consideración de } \\
\text { imparcialidad. }\end{array}$ & Ausencia de Test. \\
\hline Psicólogo Forense & -Formación especializada. & $\begin{array}{l}\text {-Regulación legislativa de las } \\
\text { condiciones de acceso a la } \\
\text { práctica forense. }\end{array}$ & $\begin{array}{l}\text {-Formación estandarizada por } \\
\text { la Administración Pública. }\end{array}$ & $\begin{array}{l}\text {-Pluralidad de profesionales. } \\
\text { Quejas de los letrados por los } \\
\text { firmantes de los informes. }\end{array}$ \\
\hline $\begin{array}{c}\text { Evaluación de la Custodia } \\
\text { de Menores en relación a la } \\
\text { propuesta de adopción de } \\
\text { medidas }\end{array}$ & $\begin{array}{l}\text {-Evaluar a todos los miembros } \\
\text { de la unidad familiar. }\end{array}$ & $\begin{array}{l}\text {-No se debe aconsejar sobre la } \\
\text { custodia si no se puede evaluar } \\
\text { a todos los integrantes de la } \\
\text { unidad familiar. }\end{array}$ & $\begin{array}{l}\text {-Mayor facilidad para evaluar } \\
\text { a todos los miembros de la } \\
\text { unidad familiar, por ser una } \\
\text { citación judicial. }\end{array}$ & $\begin{array}{l}\text {-Se debe evaluar a todos los } \\
\text { miembros de la unidad familiar, } \\
\text { incluidos los menores. }\end{array}$ \\
\hline $\begin{array}{l}\text { Informes sobre la parentalidad } \\
\text { y estudio del menor }\end{array}$ & $\begin{array}{l}\text {-Más habitual, en particular } \\
\text { cuando el otro progenitor no } \\
\text { acude a las entrevistas. }\end{array}$ & $\begin{array}{l}\text {-Comprobar sí el menor fue } \\
\text { explorado anteriormente. } \\
\text { Evitar exploraciones } \\
\text { inadecuadas. } \\
\text { Contrastar más información } \\
\text { con terceros. }\end{array}$ & $\begin{array}{l}\text {-Más posibilidad de obtener } \\
\text { información de instituciones } \\
\text { públicas relacionadas con el } \\
\text { caso. }\end{array}$ & $\begin{array}{l}\text {-Considerar la utilización de la } \\
\text { psicometría, en su caso. }\end{array}$ \\
\hline $\begin{array}{l}\text { Valoración del Tribunal sobre } \\
\text { el informe pericial }\end{array}$ & $\begin{array}{l}\text {-Sólo un } 11 \% \text { recibieron } \\
\text { críticas negativas. }\end{array}$ & $\begin{array}{l}\text {-Menor valoración. Reticencias } \\
\text { sobre imparcialidad de algunos } \\
\text { informes. }\end{array}$ & $\begin{array}{l}\text {-Mayor credibilidad de los } \\
\text { jueces hacia los informes } \\
\text { realizados. }\end{array}$ & --- \\
\hline $\begin{array}{l}\text { Coincidencia entre las } \\
\text { recomendaciones del informe y } \\
\text { los fallos de las sentencias }\end{array}$ & $\begin{array}{l}\text {-Coincidencia mayor en dos } \\
\text { tercios de los informes. }\end{array}$ & $\begin{array}{l}\text {-Se sugiere estudiar en } \\
\text { los informes privados las } \\
\text { discordancias en futuros } \\
\text { estudios. }\end{array}$ & -Elevada coincidencia. & $\begin{array}{l}\text {-Se sugiere estudiar en } \\
\text { los informes públicos las } \\
\text { discordancias en futuros } \\
\text { estudios. }\end{array}$ \\
\hline
\end{tabular}

damente generalista del código deontológico (COP, 2010) e insuficiente información y formación especializada forense. La muestra reveló amplias diferencias en las valoraciones éticas y deontológicas en muchas situaciones hipotéticas planteadas y una considerable porción de ellos optó por soluciones contradictorias a las recomendaciones éticas (COP, 2010; EFPA, 2005).

Un estudio sobre los razonamientos judiciales en procesos de separación de 782 sentencias judiciales correspondientes al período 1993-1999 mostró que las atribuciones de la guarda y custodia determinadas, un $91.56 \%$ fueron asignadas a las madres y un $8.44 \%$ a los padres (Arce, Fariña y Seijo, 2005). Otro estudio posterior a la entrada en vigor de la Ley 15/2005 reveló un cambio de tendencia referente a la asignación de la guarda y custodia: el $71.5 \%$ de las sentencias favorables a la madre, frente a $22.5 \%$ atribuidas al padre (Novo, Quinteiro y Vázquez, 2013).

Un estudio sobre la opinión de los jueces afirmó el papel decisivo, que según los magistrados, tiene el informe psicológico para valorar los aspectos centrales sobre los que apoya la evaluación pericial (idoneidad -o mejor alternativa de custodia en derecho de familia), lo que según los autores revalida la necesidad y justificación del informe pericial, su validez, y la presencia del psicólogo en los tribunales (Aguilera y Zaldívar, 2003). De forma genérica, de acuerdo con Muñoz (sin fecha), los objetivos del informe pericial seguirán las etapas del proceso de evaluación: a) orientar sobre la opción de custodia más idónea; b) formular la hipótesis y la hipótesis alternativa; c) colegir enunciados verificables; d) contrastar las hipótesis, mediante el análisis, integración e interpretación global de resultados; e) ha de comunicar los resultados; y f) elaborar el informe psicológico pericial. Finalmente debe ser ratificado en la vista oral conforme al art. 347 de la Ley de Enjuiciamiento Civil.

Un estudio en Cataluña, (Domínguez, Gamero, González y Roca, 2009), investigó el impacto de los dictámenes del Servei d'Assessorament Tècnic en l'Àmbit de Família de Catalunya (SATAF) en los fallos judiciales, afirmando sus autores, que los resultados de sus evaluaciones tenían una importante incidencia en las sentencias.

Con el presente estudio se ha tratado de paliar la carencia de investigación, y aportar evidencia empírica directa (Symons, 2010); contribuir a aumentar el conocimiento sobre las evaluaciones de la custodia de menores, una de las áreas trascendentales de la profesión porque los efectos del divorcio recaen en los menores, en las relaciones familiares y en el desarrollo de los procesos. Elementos fundamentales para comprender la protección de la infancia, la capacidad de actuar de los progenitores en el Interés Superior del Menor, y el escenario en que se desenvuelve el divorcio (Ackerman, 2010; Echeburúa, Muñoz y Loínaz, 2011).

La escasez de investigación sobre las evaluaciones de custodia se debe, en parte, a la dificultad para acceder a los registros de los juzgados (Kelly y Ramsey, 2009) afortunadamente, 
en este caso, la cooperación institucional ha sido esencial para la investigación. La evaluación de la custodia de menores pretende afirmarse en los principios científicos de la actividad profesional. Es un deber del perito cumplir con los conocimientos contrastados en los que dicha praxis se asienta.

Actualmente no existen en nuestro país estudios sobre los informes periciales realizados por psicólogos privados e informes periciales del SATAF que analicen su relación con las sentencias judiciales, por cuanto justifica la necesidad de la presente investigación para a dar a conocer el funcionamiento actual de los informes periciales, y comprender mejor como son valorados los informes periciales por los Tribunales en las sentencias. Es necesario conocer la realidad práctica de los informes periciales forenses presentados ante los tribunales de familia en nuestro entorno.

La Administración catalana responsable del Servicio público de Asistencia Técnica a los Tribunales de Familia -SATAF- ha decidido que lo conformen profesionales con titulación de Psicología y Trabajo Social y que realicen los informes de forma indiferenciada bajo el epígrafe "informe psicosocial", luego estrictamente hablando no hay desde la perspectiva de la Administración en esta Comunidad Autónoma "informes psicológicos" sino "psicosociales". Los equipos, en cuanto a su composición, están integrados por psicólogos y trabajadores sociales, en calidad de expertos en la materia relacionada con la problemática familiar y conyugal y cumplen su tarea bajo la dependencia de los Jueces y Magistrados, con una doble función: asesoramiento previo a la resolución judicial y supervisión y control de la ejecución de esta. Por tanto, se supone que cuando intervienen y firman el dictamen son considerados especialistas.

En base a este condicionante, el elemento de análisis del presente estudio, es el informe y la comparación se realiza entre el informe pericial privado y el informe pericial del SATAF, pues interesó conocer la valoración en las sentencias judiciales sobre los informes que se presentan con contenidos psicológicos, con independencia de quien los firma.

El objetivo general de la presente investigación fue analizar la relación entre las sentencias emitidas por los magistrados/as de los juzgados de familia números $15,16,17,18,19,45$ y 51 de Barcelona y los informes periciales forenses emitidos por psicólogos privados con formación forense (PPCF), psicólogos privados sin formación forense (PPSF) y profesionales expertos del equipo psicosocial judicial (EPS).

Los objetivos específicos fueron conocer: a) las medidas propuestas en los informes periciales respecto al tipo de informe presentado sólo parentalidad, parentalidad y tipo de régimen de custodia (RC) y régimen de visitas del progenitor no custodio (RV); b) las atribuciones realizadas en las sentencias respecto al RC y RV; c) la valoración del tribunal sobre el informe pericial; d) sí el tribunal recomendó a las partes seguir las indicaciones del informe pericial; e) sí hubo acuerdo entre las atribuciones del tribunal y las recomendaciones del informe pericial sobre el RC y el RV.
En la primera hipótesis se espera encontrar diferencias significativas entre el tipo de informe y las medidas propuestas en los informes periciales de los EPS, los PPCF y los PPSF sobre el RC. En la segunda hipótesis se espera encontrar diferencias significativas entre los informes periciales de los EPS y los PP respecto de las atribuciones de las sentencias judiciales sobre el RC y RV. En la tercera hipótesis se espera encontrar diferencias significativas en la valoración del tribunal sobre el informe pericial entre los grupos EPS, PPCF y PPSF. En la cuarta hipótesis se espera encontrar diferencias significativas respecto a si la sentencia instó a las partes a seguir las recomendaciones del informe pericial; si la sentencia aludió a otros informes; si en la sentencia se citó contra informes, entre los tres grupos EPS, PPCF y PPSF. En la quinta hipótesis se espera encontrar diferencias significativas entre la sentencia sobre la atribución del RC y el RV y las recomendaciones del informe pericial del EPS, y del PP.

\section{Método}

Participantes

Se analizaron 111 informes periciales privados e informes periciales realizados por el Equip d'Assessorament Tècnic en l'Ámbit de Família (SATAF) adscrito a los juzgados de familia y las sentencias judiciales pronunciadas en primera instancia contenidas en los expedientes de divorcios contenciosos.

\section{Procedimiento y Material}

La recolección de datos se realizó mediante una plantilla confeccionada ad hoc por los autores, con una selección de ítems elaborados a través del acuerdo de expertos consultados acreditados en psicología forense por el COPC. Se realizó una prueba piloto con diez expedientes de la muestra.

Se extrajo una muestra preseleccionada de archivo que incluía informe pericial y sentencia emitida en primera instancia en el juzgado de familia. Se excluyeron otro tipo de informes que no fueran dictámenes, firmados por otro tipo de profesional, si bien, en el caso del equipo psicosocial adscrito a los juzgados los dictámenes son firmados por psicólogos y/o por trabajadores sociales. Se utilizaron 111 informes periciales, 45 privados de los cuales 26 expresaron en el dictamen, tener formación específica forense, y 19 no expresaron tener la formación específica forense y 66 informes del SATAF. En un estudio del SATAF, sus autoras, afirmaron que: “...los equipos, en cuanto a su composición, están integrados por psicólogos $y$ trabajadores sociales, en calidad de expertos en la materia relacionada con la problemática familiar y conyugal" (Domínguez et al., 2009, p. 10). De los informe del SATAF 49 fueron emitidos por psicólogos y 17 por trabajadores sociales incluidos en expedientes contenciosos, iniciados entre enero de 2007 y diciembre de 2013 en los Juzgados de Familia 15, 16, 17, 18, 19,45 , y 51 de Barcelona, y de los que se extrajo la muestra preseleccionada de archivo de expedientes contenciosos con la sentencia concluida en primera instancia, e informe pericial, entre el período de enero de 2007 a diciembre de 2013. 
El estudio obtuvo resolución favorable del Comité de Ética de Investigación de la Universidad Ramón Llull (CERURL/ 2012/001). Para el estudio de los expedientes se solicitó autorización al Tribunal Superior de Justicia de Cataluña, que la trasladó con disposición favorable para su autorización a los Secretarios Judiciales de los juzgados de familia 15, 16, 17, 18, 19, 45, y 51 de la ciudad de Barcelona.

Se trata de un estudio ex post facto retrospectivo de comparación de grupos de tipo transversal. Las variables independientes fueron los informes periciales realizados por profesionales del SATAF y/o informes efectuados por peritos privados. En este último caso se distinguió si los firmantes del dictamen informaron que poseían o si carecían de la formación específica forense. El criterio de experiencia en miembros del SATAF se estableció por haber pasado una oposición. Las variables dependientes fueron las sentencias emitidas en los juzgados de familia en expedientes contenciosos que incluían los informes periciales.

El tratamiento estadístico tuvo finalidad descriptiva para lo cual se calcularon descriptores, frecuencias y análisis $\chi^{2}$ de las variables para averiguar si habían o no diferencias significativas $(p<.05 ; p<.01 ; p<.001)$ cuando se realizaron comparaciones entre grupos.

\section{Resultados}

Características demográficas de los profesionales autores de los informes de la muestra

El $100 \%$ de los dictámenes privados fueron concluidos por psicólogos y el 74.2\% de los informes del equipo psicosocial fueron efectuados por psicólogos y el $25.8 \%$ por trabajadores sociales. Los informes privados fueron solicitados el $42.5 \%$ por la madre y el $57.5 \%$ por el padre. El 100\% de los informes del equipo psicosocial fueron ordenados por el Juez. El 13.3\% de los informes privados fueron realizados por hombres y el $86.7 \%$ por mujeres. El $10.6 \%$ de los informes del equipo psicosocial (EPS) fueron emitidos por hombres y el $89.4 \%$ por mujeres. De los 66 informes del EPS, 41 fueron firmados por un psicólogo, 7 por dos, y uno por tres psicólogos, y 17 firmados por un trabajador social. Cuando firmaron dos o tres profesionales (1 ó 2 psicólogos y un trabajador social), además de evaluar la parentalidad y la custodia exclusiva, existían alegaciones trastorno psicológico o psiquiátrico activo, y/o adopción de medidas cautelares en que se planteaba la retirada de la potestad. Todos los informes de los peritos del sector privado fueron firmados por un sólo profesional e indicaron en el informe que eran: a) un $66.7 \%$ licenciados en psicología, b) un $28.9 \%$, doctores en psicología, c) un 2.2\% especialistas en psicología clínica, y d) un $2.2 \%$ especializado en psicología infantil. En cuanto a formación de postgrado, un $35.6 \%$ de los informes privados firmados asimismo, por un sólo profesional, no manifestaron en el informe poseer formación especializada, y el 64.4\% declararon poseer alguna formación específica de máster, mediación familiar o grado de experto acreditado por el Colegio Oficial de Psicólogos.

\section{Tipo de informe pericial y adopción de medidas}

Un $73 \%$ del total de los informes periciales presentados ante los tribunales recomendaron conjuntamente el RC y el RV (Tabla 1), sin diferencias significativas entre el grupo de informes PP y el grupo de informes del EPS. En relación a la adopción de medidas, el 56.2\% del total de los informes aconsejaron la custodia exclusiva, en la que el EPS respecto del PPCF y del PPSF, obtuvo diferencias significativas $(\chi 2=15.679, \mathrm{p}<.05)$. Inversamente, los PPSF recomendaron mayor tasa de custodia compartida que los PPCF y que los EPS.

Tabla 1

Tipo de informe pericial y adopción de medidas.

\begin{tabular}{|c|c|c|c|c|c|}
\hline Tipo de informe & $\%$ Total & $\%$ Privado $^{1}$ & $\begin{array}{l}\text { \% Equipo } \\
\text { Psicosocial }^{2}\end{array}$ & $\chi^{2}$ & $p$ \\
\hline \multirow{4}{*}{$\begin{array}{l}\text {-Parentalidad } \\
\text {-Parentalidad y tipo } \\
\text { de custodia } \\
\text {-Cambio de régimen } \\
\text { de visitas }\end{array}$} & & & & \multirow[t]{4}{*}{5.2830} & \multirow[t]{4}{*}{0.71} \\
\hline & 26.1 & 15.6 & 33.3 & & \\
\hline & 73.0 & 84.4 & 65.2 & & \\
\hline & 0.9 & - & 1.5 & & \\
\hline \multirow{3}{*}{$\begin{array}{l}\text { Adopción de } \\
\text { medidas propuestas } \\
\text { en el informe }\end{array}$} & $\% \operatorname{Sin}$ & \%Con & & \multirow{7}{*}{$\begin{array}{c}\chi^{2} \\
15.679\end{array}$} & \multirow{7}{*}{$\begin{array}{c}p \\
.016^{*}\end{array}$} \\
\hline & $\begin{array}{c}\text { Formación } \\
\text { Forense }^{3}\end{array}$ & $\begin{array}{c}\text { Formación } \\
\text { Forense }^{4}\end{array}$ & $\begin{array}{l}\text { \% Equipo } \\
\text { Psicosocial }^{2}\end{array}$ & & \\
\hline & & & & & \\
\hline Ninguna & 12.5 & 23.3 & 3.1 & & \\
\hline Custodia Exclusiva & 56.2 & 53.3 & $76.6^{*}$ & & \\
\hline Custodia Compartida & 31.3 & 23.3 & 14.1 & & \\
\hline Retirada Custodia & - & - & 6.3 & & \\
\hline
\end{tabular}

Nota. 1: Psicólogo privado; 2: Equipo Psicosocial; 3: Psicólogo privado sin formación forense; 4: Psicólogo privado con formación forense. $* p<.05$

\section{Atribución del Tribunal sobre el tipo de Custodia y régimen de visitas con el progenitor no custodio}

Un $69.1 \%$ de las sentencias judiciales atribuyeron la custodia exclusiva a la madre, sin diferencias significativas respecto a los informes periciales de los PP y los EPS (Tabla 2), aunque respecto al RV, la mitad de las sentencias atribuyeron un sistema amplio de contactos paterno filiales.

Tabla 2

Atribución del Tribunal sobre el tipo de Custodia y régimen de visitas con el progenitor no custodio.

\begin{tabular}{lccccc}
\hline Atribución de custodia & \% Total & \% Privado & $\begin{array}{c}\text { \% Equipo } \\
\text { Psicosocial }^{2}\end{array}$ & $\chi^{2}$ & $p$ \\
\hline Al padre & 12.7 & 11.1 & 13.8 & 3.518 & .318 \\
A la madre & 69.1 & 71.1 & 67.7 & & \\
Compartida & 14.5 & 17.8 & 12.3 & & \\
Retirada (Instituciones) & 3.6 & - & 6.1 & & \\
Régimen de visitas & & & & & \\
Amplio & 51.6 & 60.0 & 46.4 & 3.125 & .210 \\
Estándar & 24.2 & 25.7 & 23.2 & & \\
Restricciones & 24.2 & 14.3 & 30.4 & & \\
\hline
\end{tabular}

Nota: 1: Psicólogo privado; 2: Equipo Psicosocial.

\section{Valoración del Tribunal sobre el informe pericial}

La valoración de los tribunales sobre el total de los informes periciales resultó positiva en el $56.8 \%$ del total de las sentencias emitidas (Tabla 3). Se aceptaron más los informes del EPS que los de los informes periciales privados $\left(\chi^{2}=7.257, p<.05\right)$, aunque un $36.9 \%$ de las sentencias no expresaron sus valora- 
ciones sobre los informes. No obstante, al analizar la valoración de los grupos EPS (66.7\%), PPCF (50.0\%) y PPSF (31.3\%) se hallaron diferencias significativas $\left(\chi^{2}=10.011, p<.05\right)$.

Tabla 3

Valoración del Tribunal sobre el informe pericial.

\begin{tabular}{lccccc}
\hline Tipo de valoración & \%Total & $\% \mathrm{IP}^{1}$ & \%IEPS & $\chi^{2}$ & $p$ \\
\hline Positiva & 56.8 & 42.2 & $66.7^{*}$ & 7.527 & $.023^{*}$ \\
Negativa & 6.3 & 11.1 & 3.0 & & \\
No lo expresa & 36.9 & 46.7 & 30.3 & & \\
& & & & & \\
Según nivel de formación: & & & & 10.011 & $.040^{*}$ \\
$\quad$ Sin formación específica & - & 31.3 & - & & \\
$\quad$ Positivamente & - & 6.3 & - & & \\
$\quad$ Negativamente & - & 62.5 & - & & \\
$\quad$ No lo expresa & & & & & \\
Con formación específica forense & & & & \\
$\quad$ Positivamente & - & 50.0 & $66.7^{*}$ & & \\
$\quad$ Negativamente & - & 13.3 & 3.0 & & \\
$\quad$ No lo expresa & - & 36.7 & 30.3 & & \\
\hline
\end{tabular}

Nota: 1: Informe privado; 2: Informe equipo psicosocial.

$* p<.05$

El Tribunal insta a las partes a seguir las recomendaciones del informe pericial; alude a otros informes anteriores y/o recoge contra informes en sentencia

El $50.5 \%$ de las sentencias emitidas instaron a las partes a seguir las recomendaciones del informe pericial (Tabla 4). Analizado por grupos, el EPS, respecto del PPCF y del PPSF obtuvo una diferencia significativa $\left(\chi^{2}=6.098, p<.05\right)$. Más del $88.0 \%$ de las sentencias emitidas no hicieron alusión a otros informes periciales anteriores. Los EPS lograron respecto de los PPCF y de los PPSF, una diferencia significativa $\left(\chi^{2}=9.196, p<.05\right)$. Una tasa del $92.9 \%$ de las sentencias emitidas no mencionaron contra informes periciales, sin diferencias significativas entre los grupos analizados.

\section{Tabla 4}

El Tribunal insta a las partes a seguir las recomendaciones del informe pericial; Alude a otros informes anteriores y/o recoge contra informes en sentencia.

\begin{tabular}{|c|c|c|c|c|c|c|}
\hline & \%Total & $\% \mathrm{IPF}^{1}$ & $\% \mathrm{IPSF}^{2}$ & $\%$ EPS $^{3}$ & $\chi^{2}$ & $p$ \\
\hline $\begin{array}{l}\text { Recomendación del } \\
\text { Tribunal } \\
\text { SI } \\
\text { NO }\end{array}$ & $\begin{array}{l}50.5 \\
49.5\end{array}$ & $\begin{array}{l}36.7 \\
63.3\end{array}$ & $\begin{array}{l}37.5 \\
62.5\end{array}$ & $\begin{array}{c}61.4^{*} \\
38.6\end{array}$ & 6.089 & $.048 *$ \\
\hline $\begin{array}{l}\text { Tribunal alude a otros } \\
\text { informes anteriores } \\
\text { SI } \\
\text { NO }\end{array}$ & $\begin{array}{l}11.3 \\
88.7\end{array}$ & $\begin{array}{l}25.9 \\
74.1\end{array}$ & $\begin{array}{l}14.3 \\
85.7\end{array}$ & $\begin{array}{c}3.6 \\
96.4 *\end{array}$ & 9.196 & $.010 *$ \\
\hline $\begin{array}{l}\text { Alude a contra informe } \\
\text { en sentencia } \\
\text { SI } \\
\text { NO }\end{array}$ & $\begin{array}{c}7.1 \\
92.9\end{array}$ & $\begin{array}{l}13.8 \\
86.2\end{array}$ & $\begin{array}{l}13.3 \\
86.7\end{array}$ & $\begin{array}{c}1.9 \\
98.1\end{array}$ & 5.080 & .079 \\
\hline
\end{tabular}

Nota: 1: Psicólogo privado con formación forense; 2: Psicólogo privado sin formación forense; 3 : Equipo Psicosocial.

$* p<.05$

Acuerdo/desacuerdo del informe pericial y sentencia judicial

Globalmente, el $77 \%$ del total de las sentencias judiciales mostraron acuerdo con los informes periciales (Tabla 5). Los EPS lograron una tasa superior a los PP (84.0 vs. 67.6), pero no obtuvieron diferencias significativas respecto a la recomendación del RC. Los EPS en relación al RV, lograron mayor diferencia significativa $\left(\chi^{2}=30.381, p<.001\right)$ que los PP.

Tabla 5

Acuerdo/desacuerdo del informe pericial y sentencia judicial sobre atribución de la custodia y régimen de visitas con el progenitor no custodio.

\begin{tabular}{lccccc}
\hline $\begin{array}{c}\text { Coincidencia Informe- } \\
\text { sentencia }\end{array}$ & \%Total & \%Privado & $\begin{array}{c}\text { \%Equipo } \\
\text { Psicosocial }\end{array}$ & $\chi^{2}$ & $p$ \\
\hline $\begin{array}{l}\text { Tipo de Custodia } \\
\text { Coinciden }\end{array}$ & 77.0 & 67.6 & 84.0 & 3.243 & .062 \\
No Coinciden & 23.0 & 34.2 & 16.0 & & \\
& & & & & \\
$\begin{array}{l}\text { Régimen de Visitas con } \\
\text { progenitor no custodio }\end{array}$ & & & & 30.381 & $.000^{* * *}$ \\
$\quad$ Coinciden & 61.0 & 27.5 & $81.5^{* * *}$ & & \\
$\quad$ No Coinciden & 39.0 & 72.5 & 18.5 & & \\
\hline
\end{tabular}

Nota: A pesar de haber sido presentando ante el Tribunal un informe pericial privado, el juez aludió en la sentencia al informe del Equipo Psicosocial en el $67.7 \%$ de los casos analizados.

$* * * p<.001$

\section{Discusión}

El presente estudio aporta los siguientes conocimientos sobre el informe pericial presentado en los Juzgados de Familia $15,16,17,18,19,45$ y 51, de Barcelona. Respecto de la primera hipótesis no mostraron diferencias significativas en relación a los criterios utilizados en las evaluaciones de EPS y los PPCF y los PPSF, respecto del tipo de informe pericial y de las medidas propuestas sobre el RC. Los informes periciales recomendaron conjuntamente el RC y el RV, pero en las propuestas de los EPS se decantaron más por la custodia exclusiva, respecto del PPCF y del PPSF, con diferencias significativas, en contraste, los PPSF recomendaron más la custodia compartida que los PPCF y los EPS. No se puede establecer una relación causal pero, resulta sugerente la idea de que el origen de la demanda pueda influir en las preferencias del evaluador.

Respecto de la segunda hipótesis no se hallaron diferencias significativas en relación a los criterios utilizados en las sentencias judiciales respecto del RC y RV respecto de los informes emitidos por los EPS y los PP. Las sentencias judiciales, a pesar de no mostrar diferencias significativas en relación a los informes presentados por ambos grupos, atribuyeron la custodia exclusiva (69.1 a la madre y 12.7 al padre), en una proporción muy similar a las recomendadas por los EPS (67.7), y respecto al RV, la mitad de las sentencias atribuyeron un sistema amplio de contactos paterno filiales, teniendo en consideración que eran expedientes contenciosos. El cambio legislativo del libro $2^{\circ}$ del Código Civil Catalán (Ley 25, 2010), ha propiciado un incremento de la custodia compartida y un decremento de la atribución de la custodia a la madre (Rodríguez-Domínguez, Jarne, Carbonell, 2015b). El régimen de contactos amplio atribuido en las sentencias puede estar en consonancia con la preeminencia de los derechos legales de la familia.

La tercera hipótesis mostró diferencias significativas entre los tres grupos EPS, PPCF y PPSF. La valoración de los tribunales sobre el informe pericial resultó positiva en más de la 
mitad del total de las sentencias. Las preferencias en las sentencias se decantaron por los EPS y la peor valoración recayó en los informes periciales firmados por profesionales sin formación forense. El resultado es sugerente de la mayor confianza depositada por los jueces en los EPS como figuras de soporte judicial. La complejidad de la toma de decisiones judiciales en el contexto familiar precisa de peritos especializados y formados en familia.

Respecto de la cuarta hipótesis, se observó que la mitad del total de las sentencias instaron a las partes a seguir las recomendaciones del informe pericial con una diferencia significativa a favor del EPS.

Las sentencias escasamente aludieron a otros informes anteriores, sí bien $1 / 4$ parte de los PPCF mostraron diferencias significativas. No se hallaron diferencias en las sentencias al mencionar contra informes periciales.

Respecto de la quinta hipótesis, globalmente, más de las 3/4 partes de las sentencias mostraron acuerdo con los informes EPS, PP sin diferencias significativas sobre la atribución de la custodia. Aunque, la máxima discrepancia se observó en la atribución del RV con diferencia significativa a favor del EPS. Los datos sugieren una elevada concordancia de acuerdos en el tipo de custodia, siendo mayor en los EPS. Las discrepancias pueden explicarse por la independencia de los jueces, la no vinculación del informe pericial. En relación al RV se da la mayor discrepancia con los PP e inversamente una elevada concordancia de las sentencias con los EPS, lo que puede estar en conformidad con el hecho de que los jueces solicitan específicamente informes sobre el RV a los técnicos del SATAF, ya que acostumbran a ser expedientes que requieren mayor atención por presentar más problemas para cumplir con el RV.

Es necesario tener en consideración que la Administración sostenga una situación administrativa sobre los informes periciales que cabe debatir en relación a los profesionales firmantes pues aunque la ciencia de la psicología y la del trabajo social son distintas, sus metodologías y resultados deben resultar diferentes, lo cual no es coherente con el carácter "indistinto", como bien queda evidenciado en la práctica de las pruebas psicométricas, que los psicólogos sí pueden practicarlas y los trabajadores sociales no pueden aplicarlas.

En base a nuestro estudio, vemos necesarias algunas propuestas de futuro que consisten en realizar nuevas investigaciones que faciliten el análisis del origen de las demandas y conocer las limitaciones a que están sujetas. Por otro lado sería necesario mejorar la metodología y estructuración de los informes periciales, tal como se recoge en las guías de buenas prácticas del COPC, y COPM (COPC, 2014; COPM, 2009), y lograr una mejor comprensión de las contradicciones de los peritos forenses con los principios éticos (Molina et al., 2012). Igualmente, las diferencias observadas son sugerentes de la necesidad de la regulación de la formación específica forense dada la ingente responsabilidad profesional, en concordancia con el art. $17^{\circ}$ del Código Deontológico (Colegio Oficial de Psicólogos,
2010), en dicho Código Deontológico. Además, son de especial interés subrayar los artículos $6^{\circ}, 10^{\circ}, 12^{\circ}, 15^{\circ}, 18^{\circ}, 19^{\circ}, 24^{\circ}, 25^{\circ}$, $29^{\circ}, 39^{\circ}, 41^{\circ} .42^{\circ}$. Y $48^{\circ}$, así como, el reciente proyecto no de Ley, planteado en la Comisión de Justicia de las Cortes Generales (BOCG, 2013), y por consiguiente, la formación forense puede ayudar a mejorar los informes realizados por los profesionales dedicados a ésta área.

De acuerdo con Kelly y Ramsey (2009), se ven necesarias varias cuestiones a considerar para una defensa legal del sector privado: a) convenimos con el estudio de Bow y Quinnell, (2001) en la necesidad de mejorar la calidad técnica de una buena parte de los informes periciales, especialmente de los PPSF.

Entre las limitaciones de este estudio, debemos comentar que: a) se realizó en medio natural en el ámbito aplicado por cuanto presenta mayor validez externa y menor validez interna; b) no es posible asegurar la igualdad entre los grupos respecto a otras posibles variables extrañas; c) no es posible establecer relaciones causales; ni generalizar los resultados; d) se limita a un área geográfica concreta; y e) se limita a unos juzgados especializados en Familia.

Tal como evidencia la bibliografía (Domínguez, 2014; Unicef, 2013), es muy probable que en los próximos años, los peritos forenses reciban un incremento de demandas de evaluación de la custodia de menores en casos de traslados internacionales, por lo que resulta necesario, abrir espacios específicos de formación y reflexión es ésta área relacionada con los derechos de los menores (Rodríguez-Domínguez, Jarne y Carbonell, 2015a).

Son precisos nuevos estudios que permitan comparar los resultados obtenidos, porque pensamos que es la primera ocasión en que se ha realizado un estudio de esta naturaleza en nuestro país. Dadas las expectativas que suscitan estos temas, esperamos que promuevan nuevas investigaciones que ayuden a mejorar la tarea de los informes periciales emitidos por psicólogos forenses, así como su aceptación por los Tribunales.

\section{Referencias}

1. Ackerman, M.J. (2010). Essentials of forensic psychological assessment (2a. ed.). Nueva York: John Wiley y Sons.

2. Aguilera, G. y Zaldívar, F. (2003). Opinión de los jueces (derecho penal y de familia) sobre el informe psicológico forense. Anuario de Psicología Jurídica, 13, 95-122.

3. American Psychological Association. (1994). Guidelines for child custody evaluations in divorce proceedings. American Psychologist 49, 677-680. http://dx.doi. org/10.1037/0003-066X.49.7.677

4. American Psychological Association. (2009). Guidelines for Child Custody Evaluations in Divorce Proceedings. Recuperado de http://www.apa.org/practice/guidelines/ child-custody.pdf

5. American Psychological Association. (2010a). Ethical principles of psychologists and code of conduct (2002, 
Amended June 1, 2010). Retrieved from http://www.apa. org/ethics/code/index.aspx

6. American Psychological Association. (2010b). Guidelines for child custody evaluations in family law proceedings. American Psychologist, 65, 863-867. http://dx.doi. org/10.1037/a0021250

7. American Psychological Association. (2013). Specialty Guidelines for Forensic Psychology. American Psychologist, 68, 7-19. http://dx.doi.org/10.1037/a0029889

8. Arce, R., Fariña, F. y Seijo, D. (2005). Razonamientos judiciales en procesos de separación. Psicothema, 17, 57-63.

9. Boletín Oficial de las Cortes Generales. (BOCG), 342. Proposición no de Ley, 161/002019 (2013) de 15 de Octubre. Comisión de Justicia. Congreso de los Diputados.

10. Boletín Oficial del Estado. A-2010-13312. (2010). Ley 25/2010. Llibre $2^{\circ}$ Codi Civil de Catalunya.

11. Boletín Oficial del Estado. 163, 24458-24461. Ley 15/2005, de 8 de julio, por la que se modifican el Código Civil y la Ley de Enjuiciamiento Civil en materia de separación y divorcio. Recuperado de http:/www.ammediadores.es/ nueva/wp-content/uploads/2013/11/legisl fam _divorcio. pdf

12. Boletín Oficial del Estado. A-2000-323. (2000). Ley 1/2000 de Enjuiciamiento Civil.

13. Bow, J. N. y Quinnell, F. A. (2001). Psychologists' Current Practices and Procedures in Child Custody Evaluations: Five Years After American Psychological Association Guidelines. Professional Psychology: Research and Practice, 32, 261-268. http://dx.doi.org/10.1037/07357028.32.3.261

14. Colegio Oficial de Psicólogos. (2010). Código Deontológico del Psicólogo. Modificado en marzo de 2010 para su adaptación a la Ley 25/2009 de 22 de diciembre (Ley Ómnibus). Recuperado el 15 de Abril de 2015, de http:// www.cop.es/pdf/CodigoDeontologicoPsicologo-ModifAprobadaJGral13-12-14.pdf

15. Colegio Oficial de Psicólogos. (2007). Guía de actuación para psicólogos forenses. Recuperado de http://www.cop. es/pdf/GuiasDeActuacion-EnPsicologiaForense.pdf

16. Colegio Oficial de Psicólogos de Madrid. (2009). Guía de buenas prácticas para la elaboración de informes psicológicos periciales sobre custodia y régimen de visitas de menores. Recuperado de http://www.padresdivorciados.es/ wpcontent/uploads/informes-psicosociales.pdf

17. Colegio Oficial de Psicología de Catalunya. (2014). Guía de buenas prácticas para la evaluación psicológica forense y la práctica pericial. Recuperado de http://www.copc.org/ Paginas/Ficha.aspx?IdMenu=E5406E2D-6EDB-48D98026-30CCB62B7220

18. Consejo General del Trabajo Social. (2012). Código deontológico del Trabajo Social. Recuperado de http://www. cgtrabajosocial.es/codigo_deontologico

19. Del Río, C. (2005). Guía de ética profesional en psicología clínica. Madrid: Pirámide.

20. Domínguez, J.A. (2014). La ruptura de matrimonios entre españoles y extranjeros con menores. Una estadística comparada. Papers, Revista de Sociología 99, 213-234. http:// dx.doi.org/10.5565/rev/papers.593

21. Domínguez, R., Gamero, M., González, M. y Roca, A. (2009). Impacte dels informes del SATAF en les resolucions judicials. Documents de Treball. Centre d'Estudis Jurídics i Formació Especialitzada. 1-36.

22. Echeburúa, E. (2002). El secreto profesional en la práctica de la psicología clínica y forense: alcance y límites de la confidencialidad. Análisis y Modificación de Conducta, 28, 485- 501 .

23. Echeburúa, E., Muñoz, J. M. y Loínaz, I. (2011). La evaluación psicológica forense frente a la evaluación clínica: propuestas y retos de futuro. International Journal of Clinical and Health Psychology, 11, 141-159.

24. European Federation of Psychologists Associations. (2005). Meta Code of Ethics. Recuperado de http://www.efpa.eu/ ethics/ethical-codes.

25. Esbec, E. y Gómez-Jarabo, G. (2000). Psicología Forense y Tratamiento Jurídico Legal de la discapacidad. Madrid: Edisofer.

26. Horvath, L. S., Logan, T. K. y Walker, R. (2002). Child custody cases: An analysis of evaluations in practice. Professional Psychology: Research and Practice, 33, 557-565. http://dx.doi.org/10.1037/0735-7028.33.6.557

27. Illescas, A.V. (2005). Psicología del testimonio y prueba pericial. Cuadernos de Derecho Judicial, 7, 106-295.

28. Kelly, R. F., y Ramsey, S. H. (2009). Child custody evaluations: Social science child custody evaluations: The need for systems-level outcome assessments. Family Court Review, 47, 286-303. http://dx.doi.org/10.1111/j.17441617.2009.01255.x

29. Martindale, D. A., Martín, L., Austin, W. G., Drozd, L., Gould-Saltman, G., Kirpatrck, H. D. y Stahl, P. M. (2007). Model standards of practice for child custody evaluation. Family Court Review, 45, 70-91. http://dx.doi.org/10.1111/ j.1744-1617.2007.129 3.x

30. Molina, A., Arch, M. y Jarne, A. (2012). Conocimiento y Aplicación de los Principios Éticos y Deontológicos por Parte de los Psicólogos Forenses Expertos en el Ámbito de Familia. Anuario de Psicología Jurídica, 22, 767-93.

31. Muñoz, J. M. V. (sin fecha). La evaluación psicológica forense en procesos de separación y divorcio. Tribunal Superior de Justicia. Comunidad de Madrid. Recuperado de http://www.copc.org/documentos/files/seccions/ARCJUR $\%$ C3\%8DDICA $/$ jose $\% 20$ manuel $\% 20$ mu $\%$ C3 $\%$ B 1 oz. pdf

32. Novo, M., y Quinteiro, I., y Vázquez, M. J. (2013). ¿Cómo motivan los jueces la capacidad de los progenitores en las resoluciones judiciales? Anuario de Psicología Jurídica, 23, 47-51. http://dx.doi.org/10.5093/aj2013a8 
33. Rodríguez-Domínguez, C. y Carbonell, X. (2014). Coordinador de parentalidad: Nueva figura profesional para el psicólogo forense. Papeles del Psicólogo, 35, 193-200.

34. Rodríguez-Domínguez, C., Jarne, A. y Carbonell, X. (2015a). Evaluación psicológica de menores, traslados internacionales. Papeles del Psicólogo. 36, 3-13.

35. Rodríguez-Domínguez, C., Jarne, A., Carbonell, X., (2015b). Guarda y custodia de menores: razonamientos y atribuciones en las sentencias judiciales. Acción Psicológica, 12, en prensa.

36. Tippins, T. y Wittmann, J. P. (2005). Empirical and ethical problems with custody recommendations: a call for clinical humility and judicial vigilance. Family Court Review 43, 193-222. http://dx.doi.org/10.1111/j.17441617.2005.00019.x

37. Unicef. (2013). Manual del Delegado (Mayabmun 2013) Recuperado de htpp//www.anahuacmayab.mx/userfiles/ File/MANUAL\%20UNICEF...
38. Urra, J. (2007). Dilemas éticos de los psicólogos jurídicos. Anuario de Psicología Jurídica, 17, 91-109.

39. Symons, D. K. (2010). A review of the practice and science of child custody and access assessment in the United States and Canada. Professional Psychology: Research and Practice, 41, 267-273. http://dx.doi.org/10.1037/a0019271

Fecha de recepción: 11 de enero, 2015

Fecha de recepción de la $1^{\text {a }}$ versión modificada: 20 de abril de 2014

Fecha de recepción de la $2^{\mathrm{a}}$ versión modificada: 23 de mayo de 2015

Fecha de aceptación: 27 de mayo de 2015 